\section{Acute Alterations on DTI Caused by Alcohol Consumption: Region of Interest-Based Diffusion Tensor Analysis Limited Because of Poor Reproducibility, with the Additional Detrimental Effects of a Small Population Size and Minute Changes}

I read the article by Kong et al titled "Acute Effects of Alcohol on the Human Brain: Diffusion Tensor Imaging Study” in the May 2012 issue of AJNR with great interest. ${ }^{1}$ The authors reported novel findings on the world's oldest and most widely used recreational drug, ethanol, and demonstrated that it leads to some detectable alterations on DTI compared with baseline even after small to moderate amounts (for a regular drinker) of consumption. This report happens to serve as the first article in this field demonstrating DTI changes besides those well-known effects of ethanol on the CNS detectable by other tests. Their findings are extremely interesting, not only in a way linking alcohol consumption to diffusion weighted imaging (which has the same abbreviation as "driving while impaired"); but also the results presented in the article carry a potential for huge implications if validated with future studies. Nevertheless, their findings seem to be somewhat contradictory to what is known so far and extremely questionable as a result of methodologic weaknesses. I would like to address several issues regarding their DTI methodology as well as point out some objections about how the results were evaluated.

As for the methodology, apparently the authors preferred ROIbased analysis over robust automated DTI analysis, which has its own advantages and disadvantages compared with the ROI-based analysis. ${ }^{2}$ However, it is not clear why the authors used 2 different software packages for their analysis and at which step each was used, and why they switched from one software package to the other. On the basis of the images provided, it looks like they performed the analyses with FuncTool software (AW 4.3; GE Healthcare, Milwaukee, Wisconsin) and the need to use DTIStudio (Johns Hopkins University, Baltimore, Maryland) is totally unclear; expert researchers in the field would agree that even though DTIStudio is capable of coregistration to remove eddy current and motion artifacts, it is not very feasible to transfer the data back to FuncTool software, and DTIStudio also has excellent means for drawing the ROIs.

Placement of ROIs was not clearly explained. It was not clear whether these were drawn on anatomic sequences first and then copied on the ADC and fractional anisotropy maps, drawn on one of those generated maps and then copied onto other maps, or drawn separately. The last case would definitely bring additional limitations, such as reproducibility issues. The ROI size was defined as $32 \mathrm{~cm}^{2}$, except the internal capsule, for which an ROI size of $26 \mathrm{~cm}^{2}$ was used. Depending on the images provided, the ROIs should have been measured in square millimeters rather than square centimeters, and I believe if indeed that was the case, it could merely represent a typographical error in the manuscript preparation step. As a second possibility, if the ROI size represented the total cumulative size of the ROIs drawn on 1 region, then this point should have been stated in the appropriate section. However, methodologic vagueness is not the end of the story. The authors also should have explained how they reached the final values of the ROIs drawn for each area, such as whether they obtained the maximum versus minimum values for each area or averaged the values of all ROIs. Interobserver variability is a significant issue in ROI-based DTI analysis, and the authors did not clarify how the 2 observers drew their ROIs (independently versus in consensus). Absence of an interobserver variability analysis would be another confounding fact in the case of independent analysis. ${ }^{2}$

Besides the present article, only 1 other article could be identified in the literature investigating the role of DWI/DTI in acute alcohol intoxication, and this article was not cited by the authors. In their 2008 article, Duning et $\mathrm{al}^{3}$ were not able to identify any significant changes in ADC values in 4 subjects. Even though their number of subjects was smaller than that of the present study, their methodology was more consistent; for example, blood-alcohol concentrations were tabulated on the 4 occasions that the subjects underwent DWI. In the same study, the authors used a higher field magnet system, images were normalized and coregistered by using SPM2 software (Wellcome Department of Imaging Neuroscience, London, UK), and broader ROIs were traced on anatomic datasets, indicating that a more robust investigation algorithm was used with lower limitations. Their ROIs were placed to delineate the anatomic regions for more comprehensive evaluation. In the present study, even though the authors mentioned that the blood-alcohol levels were measured, the results were not tabulated. Hence, the statement in the conclusion that "DTI has been shown to be more effective in detecting alcoholinduced changes on the human brain compared with BAC or BrAC" is very hard to accept in the absence of any comparative tabulation. ${ }^{1}$

Both studies are subject to a common limitation: understandably, they used alcoholic beverages instead of pure ethanol. Kong et al ${ }^{1}$ used Maotai wine, which is made from fermented sorghum, whereas Duning et $\mathrm{al}^{3}$ used pure vodka. Essentially, both products are likely to contain substances besides ethanol that might show CNS effects. Marchiafava-Bignami disease is an unclear entity, initially linked to Chianti wines of Italy, but it has now has been associated with many other types of drinks. ${ }^{4}$ Nevertheless, it might still suggest an etiology in addition to ethanol.

Finally, better designed studies with greater numbers of subjects would yield more information on the acute effects of alcohol on DTI parameters. However, even in that case, instead of cytotoxic edema, as proposed by Kong et al, ${ }^{1}$ some other mechanisms would be more likely to cause the alterations that could possibly be detected on DTI. Alcohol has been known to exert its effects through different receptors, and $\gamma$-amino butyric acid A receptors are emphasized more than the others in that regard. These are essentially ligand-gated ion channels that are potentiated by alcohol and possibly represent acute action of alcohol by causing an influx of $\mathrm{Cl}^{-}$ions, whereas $\gamma$-amino butyric acid B receptors act through $\mathrm{G}$ proteins and are responsible for more long-term effects. ${ }^{5}$ Ethanol has different types of actions on different neurotransmitter receptors; although it potentiates some, it deactivates the rest, and it is very likely that different imaging findings might be encountered depending on microstructural changes in different parts of the brain. ${ }^{5}$

\section{References}

1. Kong LM, Zheng WB, Lian GP, et al. Acute effects of alcohol on the human brain: diffusion tensor imaging study. AJNR Am J Neuroradiol 2012;33:928-34

2. Ozturk A, Sasson AD, Farrell JA, et al. Regional differences in diffusion tensor imaging measurements: assessment of intrarater and interrater variability. AJNR Am J Neuroradiol 2008;29:1124-27

3. Duning T, Kugel H, Menke R, et al. Diffusion-weighted magnetic resonance imaging at 3.0 Tesla in alcohol intoxication. Psychiatry Res 2008;163:52-60

4. Arbelaez A, Pajon A, Castillo M. Acute Marchiafava-Bignami disease: MR findings in two patients. AJNR Am J Neuroradiol 2003;24:1955-57

5. Davies $M$. The role of GABAA receptors in mediating the effects of alcohol in the central nervous system. J Psychiatry Neurosci 2003;28:263-74

Department of Radiology/Neuroradiology University of Minnesota and Hennepin County Medical Centers Minneapolis, Minnesota

http://dx.doi.org/10.3174/ajnr.A3269 